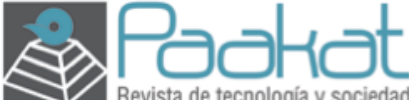

Paakat: Revista de Tecnología y Sociedad

e-ISSN: 2007-3607

Universidad de Guadalajara

Sistema de Universidad Virtual

México

paakat@udgvirtual.udg.mx

Año 11, número 20, marzo-agosto 2021

\title{
Recensión
}

\section{Fragmentar el futuro. Ensayos sobre tecnodiversidad}

\author{
Víctor Gabriel García Castañeda* \\ http://orcid.org/0000-0002-0226-3053 \\ Universitat Autònoma de Barcelona, España
}

Obra reseñada: Hui, Yuk. (2020). Fragmentar el futuro. Ensayos sobre tecnodiversidad. Buenos Aires: Caja Negra Editora.

[Recibido 05/01/2021. Aceptado para su publicación 2/2/2021]

DOI: http://dx.doi.org/10.32870/Pk.a11n20.608

En su manuscrito de 1953, La pregunta por la técnica, Martin Heidegger (1997) introdujo el concepto de Gestell -traducido comúnmente al español como "encuadre", "marco", "emplazamiento" o "aparato"- para referirse a la esencia de la técnica moderna. Heidegger percibía que, a diferencia de la concepción griega de la techné, la cual posee un carácter poiético que permite producir algo, la técnica moderna opera en el sentido contrario: esta impone -de ahí el "emplazamiento"un horizonte de comprensión o un orden que imposibilita el verdadero desocultamiento del ser. 
Heidegger realiza una crítica a este concepto de técnica debido a que implica una relación de dominación con la naturaleza al convertirla solamente en un recurso natural, en una "reserva". En este sentido, todo lo que produce la técnica moderna queda enmarcado dentro de ese modo inauténtico de relacionarnos con el mundo, lo cual, de acuerdo con el autor alemán, representa un peligro.

Tres cuartos de siglo después de la reflexión heideggeriana sobre la técnica, la tecnología contemporánea siguió el mismo curso del Gestell. Dada su completa ubicuidad, la tecnología se ha establecido como el modo primordial de relacionarnos con todo lo que nos rodea, desde nuestras relaciones personales y afectivas, hasta todo lo que sucede en los campos de la economía, la política, el arte y la propia cultura en general.

Si bien nunca ha habido aspecto humano que escape de la técnica, en el contexto actual no se trata solo del carácter antropológico universal de la misma, sino de la lógica mono-tecnológica que impone. El mundo, con toda su diversidad, parece inclinarse hacia una estandarización de los modos de relación tecnológica. Facebook, por mencionar un ejemplo, podrá alojar millones de relaciones diferentes, pero siempre enmarcadas dentro de los affordances (prestaciones) de su plataforma, la cual, a su vez, es producto de una lógica occidental que valora la tecnología en términos de progreso, eficiencia y utilidad.

Esta es la problemática a la que se enfrenta el filósofo Yuk Hui en Fragmentar el futuro. Ensayos sobre tecnodiversidad. Este libro reúne cuatro ensayos (publicados originalmente en la revista e-flux) así como tres de sus conferencias, donde Hui expone algunos de los temas que se desprenden de sus conceptos de cosmotécnica y tecnodiversidad, además de su crítica a la cultura monotecnológica. La intención de Hui, como afirma en la introducción de su libro, es la de tratar de re-enmarcar el Gestell desde un acercamiento que sea capaz de reconocer la multiplicidad de cosmologías a partir de las relaciones tecnológicas que emergen en la periferia de occidente, pero separándose al mismo tiempo de otros movimientos, como los del multiculturalismo o las críticas poscoloniales de corte más ingenuo, los cuales, a su juicio, no resuelven esta problemática. Hui plantea su proyecto bajo la forma de una antinomia:

Tesis: la tecnología es un universal antropológico; puede ser entendida como exteriorización de la memoria y liberación de los órganos, tal como lo han formulado antropólogos y filósofos de la tecnología.

Antítesis: la tecnología no es un universal antropológico; es posibilitada y constreñida por cosmologías particulares que van más allá de la funcionalidad o utilidad. Por consiguiente, no existe una única tecnología, sino múltiples cosmotécnicas (Hui, 2020, p. 11). 
Uno de los factores determinantes en la evolución humana ha sido la invención y el uso de las herramientas; desde las piedras talladas que nuestros antepasados evolutivos utilizaban como instrumentos de caza durante el Pleistoceno, hasta el reciente desarrollo de la computación cuántica. Para Hui, este fenómeno ha sido interpretado en el siglo XX por disciplinas como la filosofía, la antropología y la historia como un universal antropológico, tal como queda explícito en el pensamiento de Heidegger, pero también en el del paleontólogo francés André Leroi-Gourhan, quien consideraba a la tecnología como una extensión de los órganos y una externalización de la memoria; o la de Joseph Needham, que comparó los desarrollos tecnológicos de occidente y oriente en términos de mayor o menor avance.

Hui asevera que su intención no es la de impugnar la universalidad de la tecnología, sino la de plantear nuevas maneras de aproximarnos a ella que no necesariamente sean vistas desde la perspectiva occidental, la cual supone una desconexión de la tecnología con la realidad sobre la que se fundamenta, especialmente cuando, detrás de este deseo de universalización, se asoman los efectos del colonialismo, la modernización y la globalización, que desembocan en una cultura mono-tecnológica donde "la tecnología moderna se vuelve la principal fuerza productiva y determina en gran medida la relación entre seres humanos y nohumanos, el ser humano y el cosmos, la naturaleza y la cultura" (Hui, 2020, p. 12).

Esta cultura mono-tecnológica, imbricada profundamente en las dinámicas militares y del capital actuales, traza arbitrariamente una línea temporal -una sincronización- que se presenta como totalizante, lo cual da la ilusión de que toda la tecnología es usada de la misma manera por todos sus usuarios.

Para combatir este ímpetu dominante de sincronización y lograr re-enmarcar el Gestell, Hui propone realizar una fragmentación que "nos libere de la temporalidad histórica lineal definida por la secuencia premodernidad-modernidadposmodernidad-apocalipsis" (Hui, 2020, p. 13). Esta fragmentación, como ya advertía el mismo Hui en Recursivity and contingency (2019), no se refiere a la fragmentación asociada al pensamiento posmoderno, el cual tiene como referente un universalismo eurocéntrico y una sincronización temporal que parece llevarnos hacia la distopía del presente, sino a tratar de identificar y situar la tecnología en relación con las condiciones particulares de su ontogénesis. La apuesta de Yuk Hui es que, de romperse esta secuencia, sería posible liberar la tecnología como una herramienta del capital para convertirla en una verdadera herramienta de descolonización que permita reconocer y preservar la tecnodiversidad.

La fragmentación implica hablar de la técnica en tanto cosmotécnica, concepto que ya había adelantado en su libro The question concerning technology 
in China (2016), es decir, como una "unificación de los órdenes del cosmos y la moral a través de actividades técnicas con el propósito de sugerir que la tecnología debe ser situada en una realidad más amplia que la hace posible al mismo tiempo que la constriñe" (Hui, 2020, p. 12). De la misma manera, hablar de cosmotécnica implica reconocer la diversidad radical que existe entre sus "valores, epistemologías y modos de existencia" (Hui, 2020, p. 95), no solamente a la producción estandarizada de la misma tecnología en diferentes regiones o países, que solamente es distinguida por su marca o por características sutiles.

Estos son, a grandes rasgos, los argumentos que se encuentran de fondo en esta colección de escritos. En primera instancia, Hui despliega una crítica en contra de la interpretación del colapso de occidente como un apocalipsis mundial, producto del pensamiento sincronizado. El primero de sus ensayos demuestra la visión hegemónica occidental que interpretó los ataques del nueve de septiembre en Nueva York como la derrota del proyecto ilustrado.

Según Peter Thiel, filósofo de formación y uno de los personajes más relevantes en Silicon Valley, los ideales progresistas y liberales de la Ilustración hicieron de occidente un lugar vulnerable. Esta postura, que tiene como preocupación el declive de la civilización occidental, reaparece en los demás ensayos, por ejemplo, cuando Hui hace referencia a un artículo publicado por Henry Kissinger en The Atlantic en 2018, donde también se asegura un fracaso del proyecto ilustrado, pero en este caso a causa de la imposición de la tecnología moderna y sus históricamente inusitadas capacidades de procesamiento masivo de datos.

La preocupación por el fracaso del proyecto ilustrado occidental ha desembocado en movimientos neo-reaccionarios como el del Dark Enlightment (la Ilustración oscura), el cual rechaza el modelo democrático actual; o el aceleracionismo, particularmente en su versión más conservadora, que postula que la única manera de escapar del capitalismo es acelerar sus contradicciones para superarlo. Estas posturas no escapan de una visión de la cultura en términos monotecnológicos, pues suponen que solo existe un solo modo de relacionarnos con la tecnología, que engloba de manera homogénea a todas las sociedades por igual.

Si se habla de acelerar el colapso del capitalismo para llegar a un nuevo orden, es porque se asegura que el tecno-capitalismo ha tenido los mismos efectos en todo el mundo, cuando es evidente que mientras unos países presumen de su progreso tecnológico y las ganancias económicas de la tecnología, otros países sufren las consecuencias simbólicas y materiales de estos efectos (por ejemplo, los basureros de electrónicos en África). 
El concepto de cosmotécnica que el autor propone como modo de fragmentación proviene del concepto kantiano de cosmopolítica. Para Hui, Kant percibe una relación necesaria entre esta y la naturaleza. La única manera de llegar a una paz perpetua como modelo político es mediante la producción de una historia universal de la especie humana, y esta producción demanda un progreso de la razón que, a su vez, se presenta como telos de la naturaleza; sin embargo, el autor advierte que esta visión es universalista y no da paso a la emergencia de la tecnodiversidad. Por esto, Hui recurre al giro ontológico en la antropología -el cual asocia con autores como Bruno Latour, Tim Ingold o Philippe Descola-, que se refiere al reconocimiento de multi-naturalezas que son propias de cada localidad o región.

De acuerdo con Hui, la cosmología "es central para el concepto de 'naturaleza' y de 'ontología' de los antropólogos, ya que dicha 'naturaleza' se define según distintas 'ecologías de relaciones' en las que pueden observarse diferentes constelaciones de relaciones [...]. Estas multi-ontologías se expresan como multinaturalezas" (Hui, 2020, p. 56). Cada cultura tiene su propio modo de poiesis técnica, y sus propias epistemes y epistemologías, las cuales han quedado veladas bajo el manto de la cultura mono-tecnológica.

No obstante, el reconocimiento de multi-ontologías o de multi-naturalezas es solo el primer paso, uno que otras disciplinas como el poscolonialismo ya han dado parcialmente. Se trata, además, de intentar buscar formas de solidaridad que no se queden en lo meramente abstracto y universal, como es el caso de la paz perpetua enunciada por Kant. En cambio, es necesario buscar solidaridades concretas que respondan a problemáticas situadas, como ha sucedido en el contexto de la pandemia:

La verdadera co-inmunidad no es solidaridad abstracta, sino que parte de una solidaridad concreta cuya co-inmunidad debería servir de base para la próxima oleada de globalización (si es que la hay). Desde el comienzo de esta pandemia ha habido numerosos actos de auténtica solidaridad, en situaciones en las que resulta de suma importancia quién nos hará las compras si no podemos ir al supermercado, quién nos dará una máscara si necesitamos acercarnos al hospital, quién ofrecerá respiradores que salven vidas, etc. Hay también solidaridades entre las comunidades médicas que comparten información con vistas al desarrollo de vacunas (Hui, 2020, p. 103).

Hui retoma estas ideas de la filosofía de Gilbert Simondon, quien habla de los objetos técnicos en sus grados de abstracción y de concreción. Los objetos técnicos abstractos "son desmontables y móviles [...]. Los objetos técnicos concretos son aquellos que se cimientan (acaso literalmente) en los mundos humano y natural, entre los que actúan como un mediador" (Hui, 2020, p. 103). Bajo esta lógica, Hui considera que la forma de escapar de la abstracción 
totalizante del Gestell es al fomentar tecnodiversidades concretas. Si el capitalismo voraz mono-tecnológico deviene en la crisis climática y la explotación de los recursos naturales, la tecnodiversidad representa, precisamente, la alternativa de introducir modos diversos de relacionarnos con la tecnología fuera del Gestell tecno-capitalista.

Hui se pregunta, además, sobre el papel que tienen o tendrán las máquinas en este mundo y su ecología. Más que tratar de seguir la postura organicista que tanto se ha planteado en la tecnología -donde se trata de equiparar, al modo de la cibernética, los sistemas tecnológicos con los sistemas naturales o biológicos-, el autor afirma que no se trata de introducir la máquina a la ecología, sino de configurar una ecología de máquinas, lo cual implicaría apelar también a la importancia de la tecnodiversidad en este mundo.

\footnotetext{
El fundamento de la ecología son las diversidades, ya que solo a partir de las biodiversidades (la variedad y variabilidad de genes, especies y ecosistemas) es posible conceptualizar el sistema ecológico. Para discutir la ecología de las máquinas necesitamos un concepto análogo al de biodiversidad: la tecnodiversidad. La biodiversidad es a su vez un correlato de la tecnodiversidad, ya que sin tecnodiversidad solo seremos testigos de la desaparición de las especies en manos de una racionalidad homogénea (Hui, 2020, p. 130).
}

Hui utiliza como ejemplo el uso de pesticidas que, si bien es eficiente a corto plazo, posteriormente afecta de manera significativa a los ecosistemas naturales; sin embargo, antes de la introducción de los pesticidas, existían diversas formas de tratar las plagas, las cuales eran más sustentables y respondían a la diversidad biológica de cada región.

El autor advierte que este énfasis reiterado en lo local no implica un regreso al etnocentrismo ni a los nacionalismos, que se basan en el modelo de políticas identitarias que hemos visto extenderse durante las últimas décadas, sino a "la capacidad de reflexionar sobre el devenir tecnológico de lo local y de evitar replegarnos a alguna forma de tradicionalismo, para que múltiples localidades puedan estar en condiciones de inventar su propio pensamiento y futuros tecnológicos" (Hui, 2020, p. 131). Por lo tanto, no se trata de ligar la producción técnica con alguna tradición étnica situada en un territorio delimitado, sino de descubrir las diferentes iteraciones de la técnica en contextos no colonizados por el Gestell y sus ideologías. Como buen alumno de Gilbert Simondon, Hui evita hablar del ser humano como dominante o dominado de la tecnología, particularmente cuando la técnica se utiliza como un instrumento de poder, es decir, como una herramienta tecnocrática. 
El libro concluye con un ensayo sobre los límites de la inteligencia artificial en el contexto actual. Comúnmente se piensa que los sistemas inteligentes, como los ordenadores y sus algoritmos, ya han superado las capacidades de los humanos y, por lo tanto, estos últimos serán reemplazados por las máquinas eventualmente. Hui hace una crítica a esta postura que percibe la inteligencia solo como un procesamiento de información y menciona que, en cambio, es necesario incluir en ella los modos no-racionales de relacionarnos con el mundo (como sucede con el arte y la religión). La inteligencia artificial ofrece la posibilidad de construir una noodiversidad que nos permita pensar y entender de forma distinta al mundo, pero esto solo será posible en la medida en que se abra paso a la tecnodiversidad en esta relación.

Fragmentar el futuro. Ensayos sobre tecnodiversidad ofrece una visión que sortea la dicotomía del pesimismo-optimismo respecto a los discursos coloquiales con los que se concibe la tecnología en el presente: no es el mal de todos los males ni la salvación esperada. De acuerdo con Simondon, es el ser humano el que debe fungir como soporte de las máquinas y su organización. Por lo anterior, Hui destaca de forma reiterada en la necesidad de encontrar en las máquinas nuevos tipos de relación no estandarizada, que nos permitan habitar un mundo futuro que escape del Gestell mono-tecnológico que el capitalismo y el persistente colonialismo nos imponen en el presente.

\section{Bibliografía}

Heidegger, Martin (1997). La pregunta por la técnica, en Martin Heidegger, Filosofía, ciencia y técnica. Santiago de Chile: Editorial Universitaria.

Hui, Yuk. (2020). Fragmentar el futuro. Ensayos sobre tecnodiversidad. Buenos Aires: Caja Negra Editora.

Hui, Yuk. (2019). Recursivity and Contingency. Londres: Rowman \& Littlefield.

Hui, Yuk. (2016). The Question Concerning Technology in China. An Essay in Cosmotechnics. Falmouth: Urbanomic. 
Este artículo es de acceso abierto. Los usuarios pueden leer, descargar, distribuir, imprimir y enlazar al texto completo, siempre y cuando sea sin fines de lucro y se cite la fuente.

\section{CÓMO CITAR ESTE ARTÍCULO:}

García Castañeda, V. G. (2021). Recensión. Fragmentar el futuro. Ensayos sobre tecnodiversidad. Paakat: Revista de Tecnología y Sociedad, 11(20). http://dx.doi.org/10.32870/Pk.a11n20.608

* Estudiante del Doctorado en Filosofía en la Universitat Autònoma de Barcelona y profesor de asignatura en la Universidad Iberoamericana de la Ciudad de México. 